Revista Latinoamericana de la Papa 23 (2): 28 - 38, 2019

ISSN: 1853-4961

http://ojs.papaslatinas.org/index.php/rev-alap/index

\title{
Utilización de antioxidantes en papa (Solanum tuberosum L) mínimamente procesada
}

\author{
L.M. Garcia-Procaccini ${ }^{1 / *}$; S.B. Capezio ${ }^{2}$
}

Recibido: 16/11/2019

Aceptado: 24/11/2019

Accesible en línea: Diciembre 2019

\section{Resumen}

La papa (Solanum tuberosum L.) es una hortaliza con un destacado valor nutricional que se puede comercializar como mínimamente procesada. La vida útil de estos productos es disminuida por la reacción de pardeamiento enzimático. La concentración elevada de compuestos fenólicos, otorgan actividad antioxidante, a la vez que son sustratos de las enzimas responsables del pardeamiento enzimático. El objetivo de este trabajo fue analizar el contenido de fenoles y el pardeamiento enzimático de productos mínimamente procesado de tres variedades de papa utilizando un tratamiento antioxidante (ácido ascórbico y ácido cítrico) y un tratamiento control, durante 15 días de almacenamiento a $4^{\circ} \mathrm{C}$. Innovator, fue la variedad que menor cambio de color presentó durante el almacenamiento, aunque se caracterizó por un bajo contenido de fenoles totales. Newen INTA fue la que mejor respondió al tratamiento con antioxidantes, logrando mantener por 15 días de almacenamiento productos totalmente aceptables, sin notables cambios de color. Spunta, fue la variedad que mayor contenido de fenoles totales presentó, sin mostrar un perceptible cambio de color durante su almacenamiento con el agregado de antioxidantes.

Palabras clave adicionales Variedades de papa, fenoles totales, cambio de color, ácido cítrico, ácido ascórbico.

\section{Use of antioxidants in minimally processed potato \\ Summary}

Potato (Solanum tuberosum L.) is a vegetable with an important nutritional value that can be marketed as minimally processed. The life of these products is reduced by enzymatic browning. The high concentration of phenolic compounds gives antioxidant activity, while they are substrates for the enzymes responsible for enzymatic browning. The objective of this work was to analyze the total phenol content and the enzymatic browning of minimally processed products of three potato varieties using an antioxidant treatment (ascorbic acid and citric acid) and a control treatment, during 15 days of storage at $4 \mathrm{C}$. Innovator, was the variety that showed the least color change during storage, although it was characterized by a low content of total phenols. Newen INTA was the one that best responded to the antioxidant treatment, keeping totally acceptable products for 15 days, without perceptible color changes. Spunta was the variety with the highest total phenolic content, without showing a noticeable color change during storage with the addition of antioxidants.

\footnotetext{
* Autor para correspondencia. E-mail: luzgarciaprocaccini@yahoo.com

${ }^{1}$ Ayudante Graduada. Facultad de Ciencias Agrarias UNMdP. Ruta 226 km. 73,5 Balcarce, Argentina.

${ }^{2}$ Profesor Asociado, Facultad de Ciencias Agrarias UNMdP. Ruta 226 km. 73,5 Balcarce, Argentina.
}

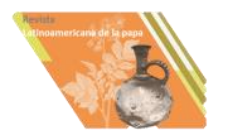


Additional key words. Potato varieties, total phenols, color change, citric acid, ascorbic acid.

\section{Introducción}

La papa (Solanum tuberosum L.) es un alimento básico para los seres humanos, y es el tercer cultivo a nivel de consumo más importante después del arroz, el trigo y el maíz (Devaux et al., 2014). La papa está compuesta por carbohidratos (almidón y azúcares solubles), proteínas, lípidos, minerales, ácidos orgánicos, compuestos fenólicos, vitaminas y agua.

Los efectos positivos de la papa que promueven la salud dependen de muchos antioxidantes naturales como polifenoles, carotenoides, tocoferoles, ácido ascórbico y selenio (Hamouz et al., 2007). El contenido total de compuestos fenólicos es una característica de cultivar (Méndez et al., 2004), pero puede ser modificado también por el ambiente y el manejo postcosecha (Akyol et al., 2016).

En los últimos años en Argentina se ha incrementado el consumo de papa mínimamente procesada que consiste en pelar y cortar los tubérculos en bastones o cubos, envasarlos y almacenarlos a temperatura de $4-5{ }^{\circ} \mathrm{C}$ hasta su venta. La mayor dificultad de estos productos es su corta vida útil debido a las alteraciones sensoriales, microbiológicas y químicas. En el tejido cortado, en presencia de oxígeno, se origina un pardeamiento enzimático causado por la acción de la enzima polifenoloxidasas (PPO) sobre los compuestos fenólicos, que provoca la formación de pigmentos oscuros. Esta enzima puede inactivarse mediante el uso de calor, inmersión en ácido cítrico o antioxidantes. La inactivación con calor es muy ineficaz ya que modifica las características sensoriales del producto (Gravoueille, 1999). Distintos autores (Ahvenainen, 1996; García y Barrett, 2002) sugieren la utilización de compuestos químicos para retrasar el pardeamiento, tales como agentes reductores, acidulantes, quelantes y acomplejantes. En la industria existe un uso extendido de los sulfitos como agentes antioxidantes. Los sulfitos pueden provocar asma (Peroni y Boner, 1995) y sabores desagradables así como una significativa reducción del valor nutricional de las papas IV Gama o mínimamente procesadas, lo que ha motivado numerosas investigaciones tendientes a encontrar un sustitutivo eficiente a estos compuestos. Diferentes inhibidores del pardeamiento han sido investigados para reducir el pardeamiento de frutas y hortalizas tales como ácido cítrico, ácido ascórbico, ácido pirofosfato de sodio, compuestos de sulfidrilo (Gunes y Lee 1997; Rocculi et al., 2007).

Algunas variedades de papa son más susceptibles a ennegrecer que otras debido al contenido de polifenoles por lo cual no estarían adaptadas a elaboración de productos mínimamente procesados y su uso indebido daría lugar a productos de baja calidad que causan insatisfacción del consumidor. Thybo et al. (2006) hallaron un efecto del genotipo sobre las características sensoriales y compuestos químicos que inducen al pardeamiento enzimático de la papa tales como el ácido clorogénico, la tirosina y la actividad de la PPO. Asimismo Cabezas-Serrano et al. (2009) evaluando 5 variedades de papa, encontraron que la variedad Agata y Marabel tuvieron menor susceptibilidad al pardeamiento, debido al bajo contenido de fenoles y a la baja actividad de la PPO, al ser comparadas con Agria, Almera y Vivaldi.

En Argentina el mercado en fresco está dominado por la variedad Spunta y es la que generalmente se utiliza como mínimamente procesado. Sin embargo 
existen otras variedades que no han sido suficientemente evaluadas. Por lo cual el objetivo de este trabajo fue analizar el contenido de fenoles y el pardeamiento enzimático de tres variedades de papa utilizando un tratamiento antioxidante que consistió en una combinación de ácido ascórbico y ácido cítrico.

\section{Materiales y Métodos}

Se utilizaron tubérculos provenientes de dos ensayos a campo plantados en un lote en la Unidad Integrada Balcarce (EEA INTA- Fac. Cs Agrarias UNMdP, $37^{\circ} 45^{\prime}$ 32 ' S; 58 $8^{\circ}$ ' $17^{\prime}$ 'O) en octubre de 2015 y 2016 que siguieron un diseño en bloques completos aleatorizados con tres repeticiones. Se utilizaron tres variedades Innovator, Newen INTA y Spunta. El manejo del cultivo en los dos ensayos fue el tradicional de la zona. La cosecha se realizó cuando los tubérculos alcanzaron la madurez fisiológica, en abril de 2016 y en marzo 2017 respectivamente. Los tubérculos fueron almacenados en una cámara a $7{ }^{\circ} \mathrm{C}$ hasta su utilización. Se tomó una muestra de $20 \mathrm{~kg}$ de cada variedad y repetición y los tubérculos fueron lavados primeramente con agua corriente $y$ un segundo lavado con hipoclorito de sodio (200 ppm - 3 minutos). Luego se pelaron y cortaron los tubérculos en bastones de $1 \mathrm{~cm} \times 1 \mathrm{~cm} x$ el largo de cada variedad, con una bastonera. Posteriormente se aplicó a la mitad de la muestra un tratamiento antioxidante (1\% ácido ascórbico $+1 \%$ ácido cítrico) por inmersión en una relación producto: solución (1:3) durante 3 minutos con agitación manual y se eliminó el exceso de solución de los bastones a través de un tamiz. La otra mitad de los bastones (testigo) se enjuagaron con agua corriente. Se utilizaron bolsas de polietileno de baja densidad de $70 \mu \mathrm{m}$ para colocar los bastones $(300 \mathrm{~g})$ de ambos tratamientos.
Las bolsas se almacenaron a $4{ }^{\circ} \mathrm{C}$ durante 15 días. $\mathrm{Al}$ inicio, a los 5, a los 10 y a los 15 días del almacenamiento se tomaron 3 bolsas de cada tratamiento y variedad para su análisis.

Se estimó el pardeamiento enzimático y el contenido de fenoles totales tomando 5 bastones al azar de cada muestra. Para la estimación del pardeamiento enzimático se empleó un colorímetro triestímulo Minolta modelo CR300 usando la representación en tres dimensiones CIE 1976 L*: luminosidad, a*: enrojecimiento (rojo-verde) y b*: amarillamiento (azulamarillo) (Whitaker y Lee, 1995). A partir de los tres dimensiones $\mathrm{L}^{*}, \mathrm{a}^{*}, \mathrm{y} \mathrm{b}^{*}$ se calculó el índice de pardeamiento (IP) y el cambio total de color $(\Delta \mathrm{E})$. El índice de pardeamiento se calculó mediante la ecuación (1), que representa la pureza del color marrón y es considerado como un importante parámetro asociado con el pardeamiento (Ali et al., 2016).

$\mathrm{IP}=(100 \mathrm{x}(\mathrm{X}-0,31)) / 0,17$

Donde:

$X=\left(a^{*}+1,75 \times L^{*}\right) /\left(5,645 \times L^{*}+a^{*}-3,012 \times b^{*}\right)$. El $\Delta$ E se calculó como la $\left(\Delta \mathrm{L}^{* 2}+\Delta \mathrm{a}^{* 2}+\Delta \mathrm{b}^{* 2}\right)^{1 / 2}$

El contenido de fenoles totales se determinó por el método espectrofotométrico de Folin-Ciocalteu descripto por Blessington (2005) con modificaciones. Se realizó una curva de calibración de ácido gálico y los resultados se expresaron como $\mu \mathrm{g}$ de ácido gálico equivalente (AGE) por gramo de peso fresco.

Se realizaron análisis de varianza y de correlación por año, día de almacenamiento y por variedad. Se realizaron análisis de media cuando se detectaron diferencias significativas. Para todos los análisis se utilizó el paquete estadístico R (2015). 


\section{Resultados y Discusión}

\section{Contenido de fenoles}

En las figuras 1 y 2 se presenta el contenido de fenoles totales ( $\mu \mathrm{g}$ AGE/g pf) de productos minimamamente procesados de tres variedades de papa con y sin aplicación de antioxidantes al momento del corte y a los 5, 10 y 15 días de almacenamiento a $4^{\circ} \mathrm{C}$ para 2016 y 2017.

La aplicación de antioxidantes aumentó el contenido de fenoles totales en las tres variedades evaluadas en los dos años de ensayo a largo del almacenamiento con respecto al testigo. Se hallaron diferencias significativas $(\mathrm{p}<0.01)$ entre las variedades y entre los tratamientos en el momento del corte en los dos años de ensayo. Hubo interacción significativa entre años, variedad y tratamiento. Innovator presentó valores significativamente más bajos que Spunta y Newen en los dos años para los dos tratamientos. Sin aplicación de antioxidantes los valores fueron significativamente diferentes en los dos años (274 y $336 \mu \mathrm{g}$ AGE/g pf en 2016 y 2017 respectivamente), lo cual indica que las condiciones ambientales durante el desarrollo del cultivo afectaron el contenido de fenoles. Con la aplicación de antioxidantes el valor aumentó a 425 y $595 \mu \mathrm{g}$ AGE/g pf respectivamente. Los bastones de Newen sin antioxidantes presentaron valores más bajos que Spunta en el 2016 pero no se diferenciaron de ésta en el 2017. En cambio con la aplicación de antioxidantes no hubo diferencias significativas entre las dos variedades en los dos años de ensayo. Cabezas Serrano et al. (2009) evaluando 5 cultivares de papa para ser procesados como productos recién cortados, también demostró gran variabilidad entre ellas, tanto en su contenido de fenoles como en los cambios de color durante el almacenamiento. En su trabajo, la variedad Almera mostró el mayor contenido de fenoles (461 $\mu \mathrm{g}$ AGE / gpf), mientras que el más bajo fue en la variedad Marabel (327 $\mu \mathrm{g}$ AGE / gpf). Otros trabajos también demostraron que el contenido de fenoles varía entre cultivares (Thybo et al., 2006; Mattila and Hellström, 2007).

A lo largo del almacenamiento se observó una disminución no significativa en el contenido de fenoles en las tres variedades siendo un poco más acentuado en el 2017 (Fig. 1 y 2).

Varios trabajos sostienen que el contenido de nutrientes de la papa, y en particular de los fenoles está influenciado por una serie de factores, siendo la variedad la más importante. Andre et al. (2007) observaron una diferencia de once veces en el contenido de fenoles totales entre variedades de papa andina. Navarre et al. (2009) encontraron una diferencia de quince veces en su contenido fenólico, evaluando un total de 100 genotipos. Un procesamiento mínimo como es el manejo, el lavado y el corte puede causar cambios en los fitoquímicos y puede conducir a la activación de algunas enzimas que modifican el nivel de compuestos fenólicos (Tudela et al., 2002). Aunque la PPO se considera un factor importante, los niveles altos de fenoles, demostraron tener una influencia positiva en el pardeamiento de los tubérculos de papa (Corsini et al., 1992; van Eck, 2007). 


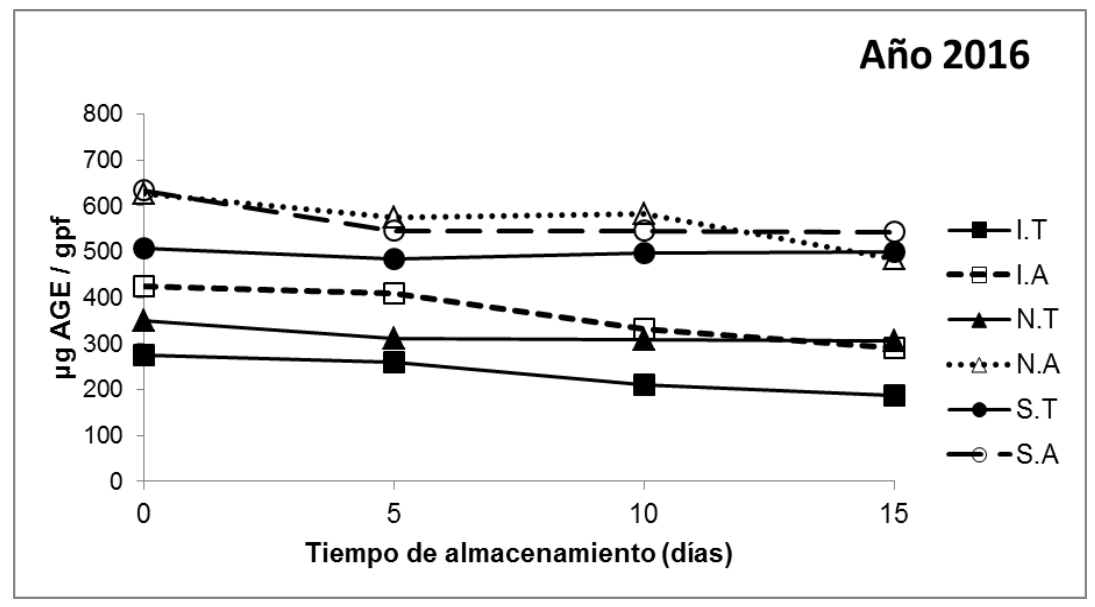

Figura 1. Contenido de fenoles totales (ug AGE/g pf) en pmp de tres variedades de papa con y sin aplicación de antioxidantes en almacenamiento. Balcarce, 2016. I.T: Innovator.Testigo, I.A: Innovator.Antioxidante; N.T: Newen.Testigo, N.A: Newen.Antioxidante, S.T: Spunta.Testigo, S.A: Spunta.Antioxidante.

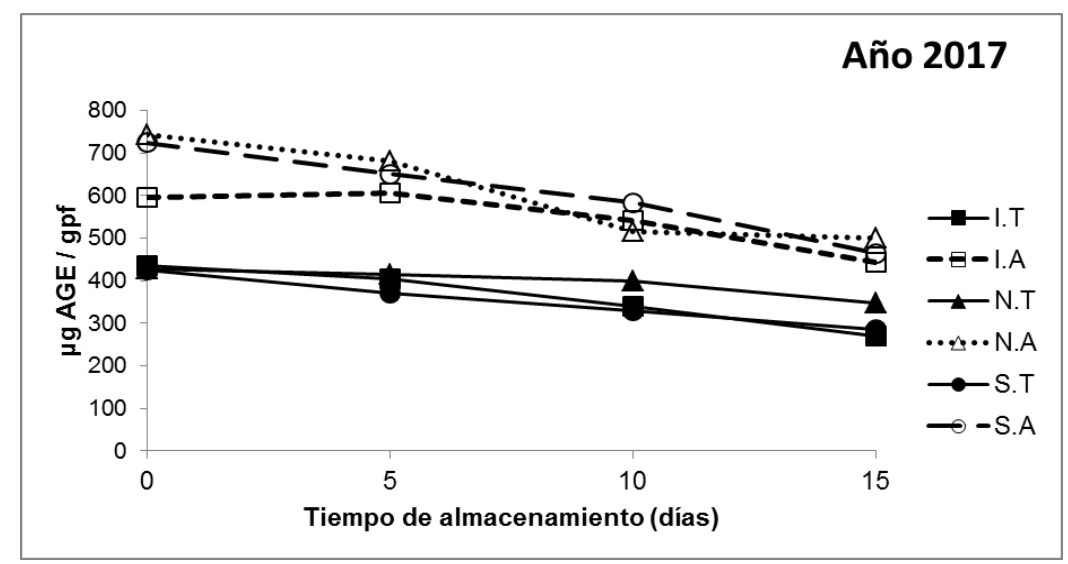

Figura 2. Contenido de fenoles totales (ug AGE/g pf) en pmp de tres variedades de papa con y sin aplicación de antioxidantes en almacenamiento. Balcarce, 2017

I.T: Innovator.Testigo, I.A: Innovator.Antioxidante; N.T: Newen.Testigo, N.A: Newen.Antioxidante, S.T: Spunta.Testigo, S.A: Spunta.Antioxidante.

\begin{tabular}{|c|c|}
\hline del pardeamiento & $\begin{array}{l}\text { colorimétricos } \mathrm{L}^{*}, \mathrm{a}^{*} \text { y } \mathrm{b}^{*} \text {. Se encontró } \\
\text { diferencia en el IP entre variedades en el }\end{array}$ \\
\hline $\begin{array}{l}\text { El IP es considerado una medida } \\
\text { importante del color marrón desarrollado } \\
\text { como consecuencia del pardeamiento } \\
\text { enzimático en el tejido de vegetales } \\
\text { frescos (Palou et al., 1999). Este } \\
\text { involucra los tres parámetros }\end{array}$ & $\begin{array}{l}\text { día } 0 \text { y durante el almacenamiento. } \\
\text { Newen INTA presentó mayor IP que las } \\
\text { otras variedades en los bastones sin tratar. } \\
\text { Con la aplicación de antioxidantes esta } \\
\text { variedad mejora su comportamiento no } \\
\text { presentando signos de pardeamiento (Fig } \\
3 \text { y 4). }\end{array}$ \\
\hline
\end{tabular}


A medida que aumenta el tiempo de almacenamiento, aumenta el $\Delta \mathrm{E}$, lo que implica que el oscurecimiento de los bastones se hace perceptible. En Newen INTA la diferencia de cambio de color entre el testigo $y$ el tratado con antioxidante es mayor que en el resto de las variedades.

En las muestras testigos (PMP sin tratar), en el 2016 se encontró una correlación positiva y significativa para la variedad Innovator entre el contenido de fenoles y el valor L*. No así en Spunta y Newen que mostraron una correlación negativa y significativa entre ambos parámetros. En el año 2017, Spunta presentó una correlación positiva y significativa entre el contenido de fenoles totales y el IP, mientras que Innovator dio una correlación negativa.

En las muestras tratadas con antioxidantes, en el año 2016, los PMP de las variedades Innovator y Spunta mostraron una correlación positiva entre el contenido de fenoles totales y el valor L*. Mientras que en el año 2017, las tres variedades mostraron una correlación positiva entre el contenido de fenoles y el valor $\mathrm{L}^{*}, \mathrm{y}$ por consiguiente una correlación negativa entre los fenoles y el IP.

Se encontraron grandes diferencias en términos de parámetros colorímetros durante el almacenamiento de las tres variedades de papa utilizado en este experimento, lo cual coincide con lo obtenido por otros autores (Mattilia et al., 1993; Gorny et al., 1999).

Estos resultados pueden explicarse con las diferencias observadas en los fenoles

totales entre las variedades. Cabezas Serrano et al. (2009) en su trabajo, también encontró diferencias en cultivares de papa durante su almacenamiento a $5^{\circ} \mathrm{C}$, correlacionando un mínimo cambio de color con un bajo contenido de fenol y actividad de PPO. No todos los fenoles tienen la misma afinidad con las enzimas PPO. Algunos autores, de hecho, encontraron una correlación positiva entre el contenido de fenoles con el pardeamiento enzimático (Friedman, 1997; Thybo et al., 2006), mientras que en otras variedades encontraron una correlación negativa entre el ácido cafeico y la decoloración enzimática en papas. Por otro lado, Cabezas Serrano et al., (2009) mostró que Almera tuvo el peor rendimiento post corte, y lo relacionó con su alto contenido de enzima y contenido de fenoles. 


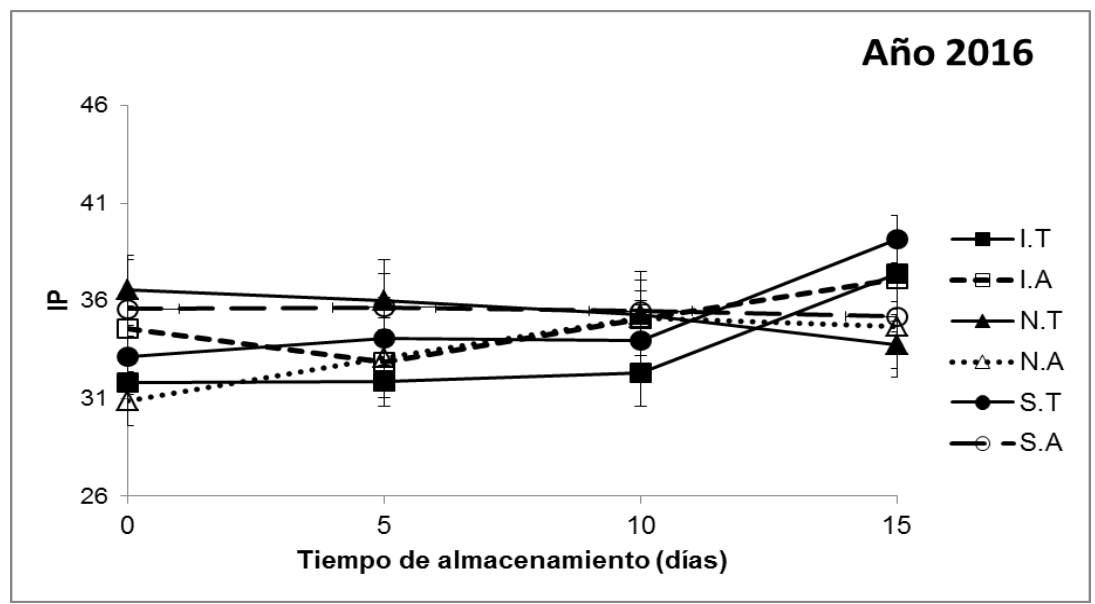

Figura 3. Indice de pardeamiento (IP) en pmp de tres variedades de papa con y sin aplicación de antioxidantes en diferentes momentos de almacenamiento. Balcarce, 2016

I.T: Innovator.Testigo, I.A: Innovator.Antioxidante; N.T: Newen.Testigo, N.A: Newen.Antioxidante, S.T: Spunta.Testigo, S.A: Spunta.Antioxidante.

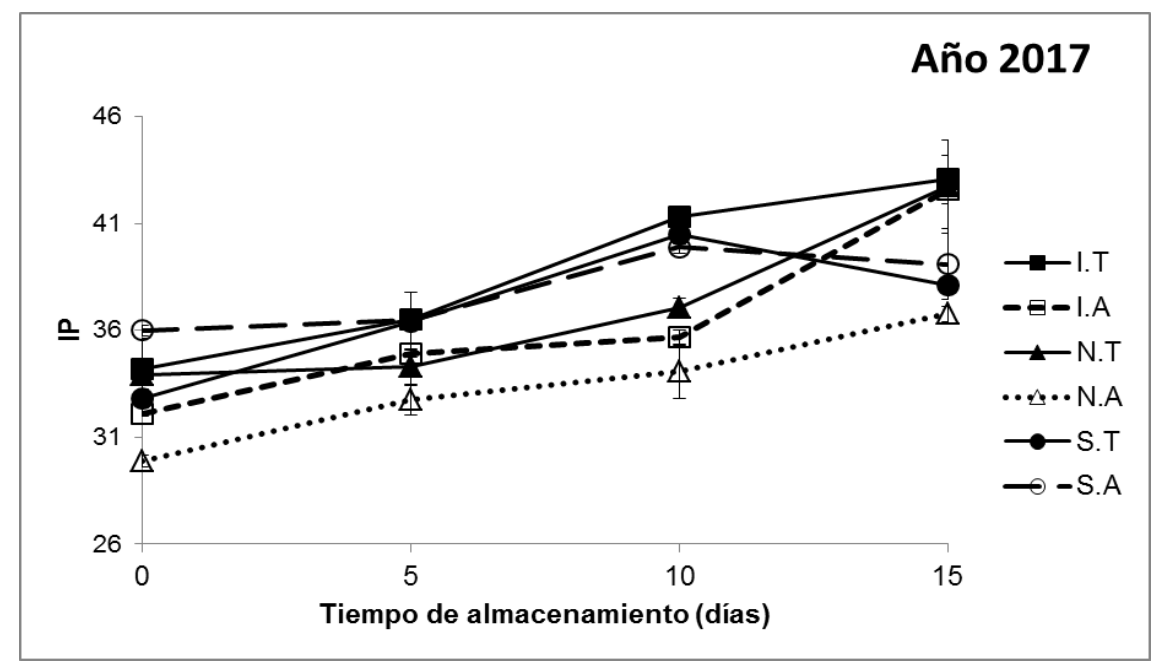

Figura 4. Indice de pardeamiento (IP) en pmp de tres variedades de papa con y sin aplicación de antioxidantes en diferentes momentos de almacenamiento. Balcarce, 2017

I.T: Innovator.Testigo, I.A: Innovator.Antioxidante; N.T: Newen.Testigo, N.A: Newen.Antioxidante, S.T: Spunta.Testigo, S.A: Spunta.Antioxidante.

\section{Cambio total de color}

En las Fig. 5 y 6 se muestra el cambio total de color de los PMP de papa de las 3 variedades con $\mathrm{y}$ sin tratar durante el almacenamiento a $4^{\circ} \mathrm{C}$.
Siguiendo el criterio empleado por Limbo y Piergiovanni (2006), el cambio de color durante un periodo de tiempo puede ser considerado perceptible ( $\triangle \mathrm{E}: 3-6)$ y fuertemente perceptible ( $\Delta \mathrm{E}: 6-12)$ en el resto de los tratamientos. 
Para la variedad Newen, el cambio total de color es significativamente diferente entre los PMP de papa tratados y los sin tratar. Sin la aplicación de antioxidantes mostró un $\Delta \mathrm{E}$ superior que el resto de las variedades en los dos años ensayados. Por lo cual el uso de antioxidantes en esta variedad dio un resultado satisfactorio.

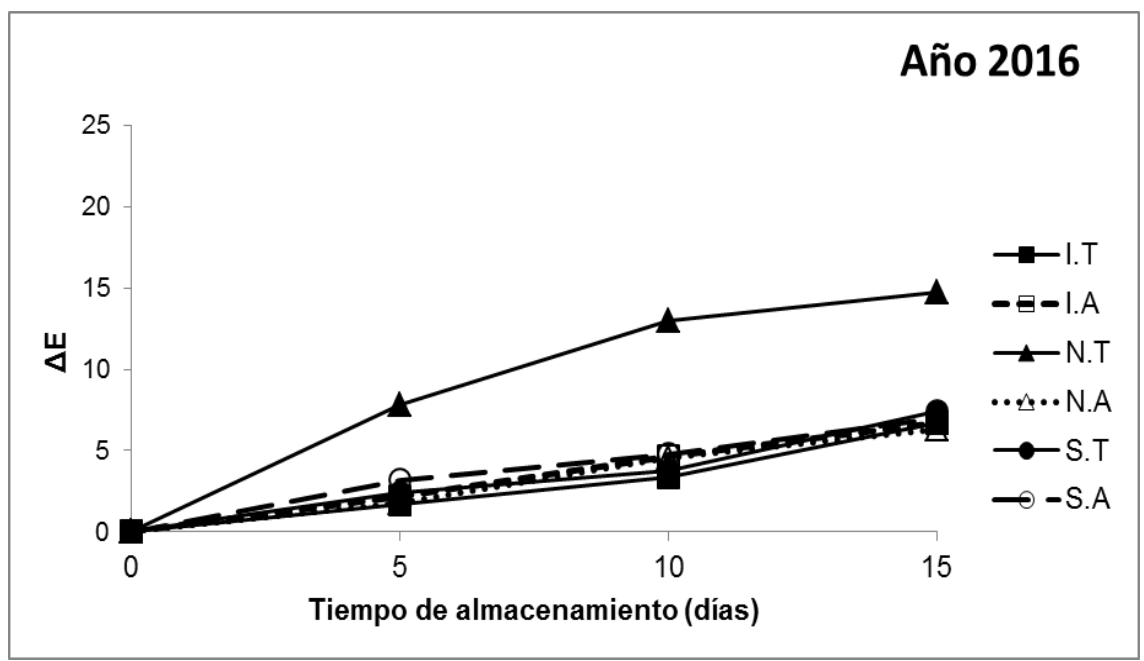

Figura 5. Cambio total de color $(\Delta \mathrm{E})$ en pmp de tres variedades de papa con y sin aplicación de antioxidantes en diferentes momentos de almacenamiento. Balcarce, 2016

I.T: Innovator.Testigo, I.A: Innovator.Antioxidante; N.T: Newen.Testigo, N.A: Newen.Antioxidante, S.T: Spunta.Testigo, S.A: Spunta.Antioxidante.

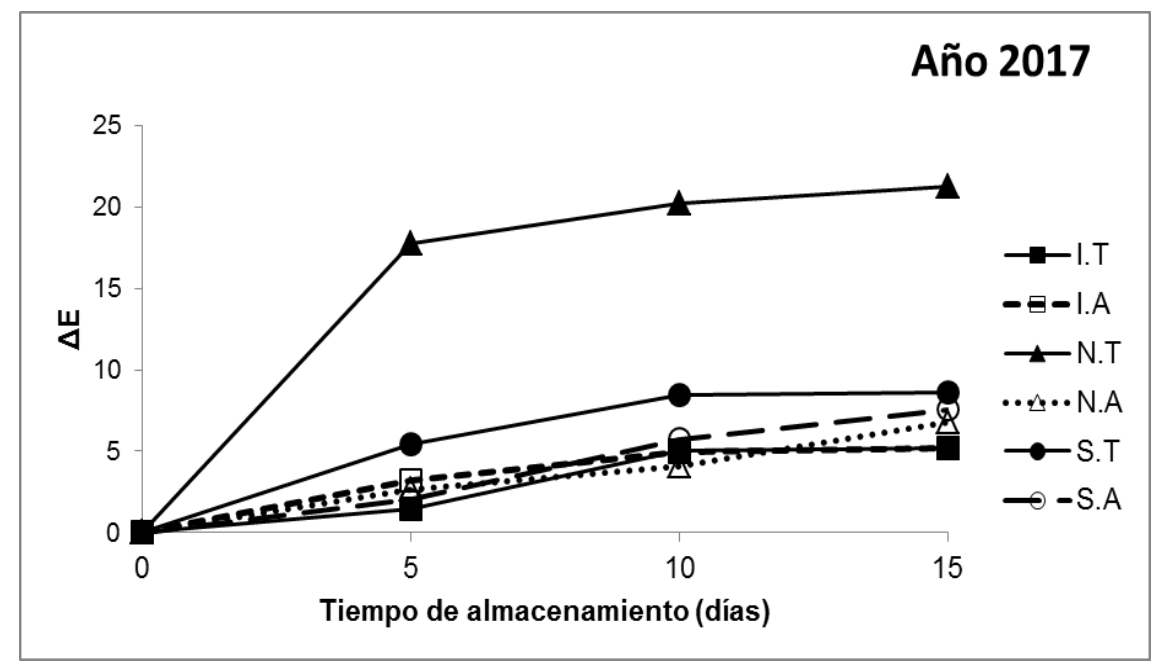

Figura 6. Cambio total de color $(\Delta \mathrm{E})$ en pmp de tres variedades de papa con y sin aplicación de antioxidantes en diferentes momentos de almacenamiento. Balcarce, 2017.

I.T: Innovator.Testigo, I.A: Innovator.Antioxidante; N.T: Newen.Testigo, N.A: Newen.Antioxidante, S.T: Spunta.Testigo, S.A: Spunta.Antioxidante. 


\section{Conclusión}

Entre los cultivares de papa ensayados en este trabajo con el fin de ser utilizados como productos mínimamente procesados, Innovator mostró menos cambios de color durante los primeros días de su almacenamiento aunque se caracterizó por un bajo contenido de fenoles, en comparación a las otras dos variedades. Newen INTA fue la variedad que mejor respondió al tratamiento con antioxidantes, logrando mantener por 15 días de almacenamiento productos totalmente aceptables, sin notables cambios de color. Spunta, fue la variedad con el mayor contenido de fenoles totales, sin mostrar un perceptible cambio de color durante su almacenamiento con el agregado de antioxidantes. Los resultados confirman las diferencias existentes entre las variedades de papa en términos de postcosecha, y la necesidad de ampliar el trabajo a otras variedades, con el fin de tener más genotipos disponibles para procesamiento.

\section{Conflictos de intereses}

La presente investigación no presenta conflicto de intereses.

\section{Agradecimientos}

Las autoras agradecen a los Sres Walter Giuliano, Jorge Ferreyra y Pablo Altamirano del INTA Balcarce por la colaboración en los ensayos realizados a campo

\section{Referencias citadas}

Ahvenainen, R. (1996). New approaches in improving the shelf life of minimally processed fruit and vegetables. Trends in Food Science \& Technology. 71: 179186.

Akyol, H.; Riciputi, Y.; Capanoglu, E.; Caboni, M.F.; Varardo, V. (2016).
Phenolic compounds in the potato and its byproducts: an overview. Int. J. Mol. Sci. 17: 835 .

Ali, H. M.; El-Gizawy, A. M.; ElBassiouny, R. E. I.; Saleh, M. A. (2016). The role of various amino acids in enzymatic browning process in potato tubers, and identifying the browning products. Food Chemistry. 192: 879-885.

Andre, C. M.; Oufir, M.; Guignard, C.; Hoffmann, L.; Hausman, J. F.; Evers, D.; Larondelle, Y. (2007). Antioxidant profiling of native Andean potato tubers (Soanum tuberosum L.) reveals cultivars with high levels of $\beta$-carotene, $\alpha$ tocopherol, chlorogenic acid, and petanin. Journal of Agricultural and Food Chemistry. 55: 10839-10849.

Blessington, T. (2005). The effects of cooking, storage, and ionizing irradiation on Carotenoids, Antioxidant Activity, and Phenolics in Potato (Solanum tuberosum L.). Tesis Master of Science. Texas A\&M University. 39-42.

Cabezas-Serrano, A.B.; Amodio, M.L.; Cornacchia B, R.; Rinaldi, R.; Colelli, G. (2009). Suitability of five different potato cultivars (Solanum tuberosum L.) to be processed as freshcut products. Postharvest Biology and Technology. 53: 138-144.

Corsini, D.L.; Pavek, J.J.; Dean, B. (1992). Differences in free and proteinbound tyrosine among potato genotypes and the relationship to internal blackspot resistance. Am. Potato J. 69: 423-435.

Devaux, A.; Kromann, P.; Ortiz, O. (2014). Potatoes for sustainable global food security. Potato Research. 57: 185199.

Friedman, M. (1997). Chemistry, biochemistry and dietary role of potato 
polyphenols. A review. J. Agric. Food Chem. 45: 1523-1540.

García, E.L.; Barrett, D.M. (2002). Preservative treatments for fresh-cut fruits and vegetables. IN: LAMIKANRA, O. (ED.), Fresh-cut fruits and vegetables. Science, Technology and Market: 267303.

Gorny, J.R.; Hess-Pierce, B.; Kader, A.A. (1999). Quality changes in fresh-cut peach and nectarine slices as effected by cultivar, storage atmosphere and chemical treatments. J. Food Sci. 64, 429-432.

Gravoueille, J.M. (1999). Utilización en la alimentación humana. En 459-505 En: Rousselle, P., Y. Robert, J. C, Crosnier (Coordinadores). La patata. Versión española de J.M. Mateo Box. Ediciones Mundi Prensa.

Gunes, G.; Lee, C.Y. (1997). Color of minimally processed potatoes as affected by modified atmosphere packaging and antibrowning agents. Journal of Food Science. 62: 572-578.

Hamouz, K.; Lachman, J.; Dvorak, P.; Duskova, O.; Cizek, M. (2007). Effect of conditions of

locality, variety and fertilization on the content of ascorbic acid in potato tubers. Plant Soil Environ. 53: 252-257.

Limbo, S.; Piergiovanni, L. (2006). Shelf life of minimally processed potatoes: Part 1. Effects of high oxygen partial pressures in combination with ascorbic and citric acids on enzymatic browning. Postharvest Biol. Technol. 39 (3): 254-264

Mattilia, M.; Ahvenainen, R.; Hurme, E. (1993). Prevention of browning of prepeeled potato. In: De Baerdemaker, J. et al. (Eds.), COST 94, Proc.Workshop "System and operation for post-harvest quality", Leuven, Belgium.
Mattila, P.; Hellström, J. (2007). Phenolic acids in potatoes, vegetables, and some of their products. J. Food Comp. Anal. 20: 152-160.

Méndez, C.D.M.V.; Delgado, M.A.R.; Rodríguez, E.M.R.; Romero, C.D. (2004). Content of free phenolic compounds in cultivars of potatoes harvested in Tenerife (Canary islands). J. Agric. Food Chem. 52: 1323-1327.

Navarre, D. A.; Goyer, A.; Shakya, R. (2009). Nutritional value of potatoes. Vitamin, phytonutrient and mineral content. In J. Singh, \& L. Kaur (Eds.), Advances in potato chemistry and technology (pp. 395-424). Austerdam: Elsevier.

Palou, E.; López-Malo, A.; BarbosaCánovas, G. V.; Welti-Chanes, J.; Swanson, B. G. (1999). Polyphenoloxidase activity and color of blanched and high hydrostatic pressure treated banana puree. Journal of Food Science. 64: 42-45.

Peroni, D. G.; Boner, A.L. (1995). Sulfite sensitivity. Clinical \& Experimental Allergy. 25: 680-681.

R CORE TEAM (2015). R: a language and environment for statistical computing. $\mathrm{r}$ foundation for statistical computing, Vienna, Austria. URL https://www.r-project.org/.

Rocculi, P.; Gómez-Galindo, F.; Mendoza, F.; Wadsö, L.; Romani, S.; Dalla Rosa, M.; Sjöholm, I. (2007). Effects of the application of antibrowning substances on the metabolic activity and sugar composition of freshcut potatoes. Postharvest biology and technology. 43: 151-157.

Thybo, A.K.; Christiansen, J.; Kaack, K.; Petersen, M.A. (2006). Effect of cultivars, wound healing and storage on sensory quality and chemical components in 
prepeeled potatoes. LWT-Food Science and Technology. 39: 166-176.

Tudela, J. A.; Cantos, E.; Espin, J. C.; Tomas-Barberan, F. A.; Gil, M. I. (2002). Induction of antioxidant flavonol biosynthesis in fresh-cut potatoes. Effect of domestic cooking. Journal of Agricultural and Food Chemistry 50: 5925-5931.

van Eck, H.J. (2007). 6.5 Tuber Quality Traits, in: Potato Biology and Biotechnology- Advances and Perspectives. Dick Vreugdenhil, Netherlands, pp. 104-115.

Whitaker, J.R.; Lee, C.Y. (1995). Recent advances in chemistry of enzymatic browning. ACS Sump. Sr. 600: 2-7. 